\title{
Pour une typologie des résumés fonctionnels
}

\author{
Céline Beaudet \\ Université de Sherbrooke
}

CETTE RECHERCHE A POUR POSTULAT de départ que la connaissance des typologies des textes et des discours ouvre des perspectives didactiques intéressantes pour l'enseignement des principes d'organisation logique profonde du texte écrit.

La saisie de ces principes se révèle en effet capitale pour la lecture minutieuse du texte et la reconstruction de sa dynamique interne, ce qui correspond à la double articulation du travail du résumeur. En examinant les diverses typologies existantes, l'apprentirésumeur découvre l'ensemble des questions par le biais desquelles il devra appréhender le texte à lire et qui lui permettront ensuite de bâtir une structure d'accueil conséquente pour la version abrégée.

Typologie, critères de classification, notions de textualité et de discursivité seront examinés ici en introduction, de manière à contextualiser l'approche retenue dans le corps de l'article, soit un développement sous forme de spirale où sont abordées tour à tour les variantes reconnues de typologie des textes et des discours. En conclusion, la démarche analytique proposée est mise en perspective par rapport aux objectifs visés dans l'enseignement du résumé fonctionnel.

\section{Typologie et lecture prédictive}

Il est démontré que la connaissance typologique est une des clés de l'efficacité en rédaction professionnelle : elle permet d'anticiper les contenus des textes à lire et d'en saisir la spécificité, comme le souligne Jean-Michel Adam lorsqu'il écrit : ( $Q$ Quels types de textes? », Le francais dans le monde, 1985, no 192) :

Il paraît possible de dire que (...) la reconnaissance des types textuels et la maîtrise de certains schémas globaux facilitent le traitement cognitif. (...) Le lecteur perçoit toujours le message le plus probable à ses yeux et lire c'est, entre autres opérations, émettre des hypothèses 
sur le type de texte.

Cette position est également soutenue par André Petitjean ( typologies textuelles ", Pratiques, no 62, juin 1989), qui écrit:

Comme cela a été abondamment vérifié pour le récit (cela reste à prouver pour les autres types de textes), il semblerait que l'organisation globale d'un texte (sa superstructure) joue un rôle fondamental dans le processus de compréhension des textes.

En d'autres termes, la connaissance du type d'écrit ou du genre de discours influe sur la lisibilité d'un texte particulier.

Par ailleurs, dans une perspective structuraliste, comme en témoigne JeanLouis Chiss (" Malaise dans la classification ", Langue française, no 74, mai 1987), la typologie des textes et des discours col̈ncide avec l'inventaire des structures abstraites: « (...) l'oeuvre apparaît (..), dans la démarche structuraliste, comme la manifestation, parmi d'autres, d'une structure abstraite. »

\section{Critères de classification}

Toute forme de typologie équivaut à une entreprise de classification derrière laquelle se profile obligatoirement une théorie organisée, avec critères et justification. Qu'est-ce donc que classer?

«Classer, comme le définit André Petitjean dans l'article déjà cité, (...) c'est opérer un rapprochement entre des objets langagiers, toujours univoques et différents, à partir de caractéristiques (propriétés) qui leur sont communes.» C'est aussi « découvrir une règle qui fonctionne à travers plusieurs textes ", comme l'écrivait à son tour Tzvetan Todorov (Introduction à la littérature fantastique, Seuil, 1970).

De son côté, A.J. Greimas (Sémiotique, Dictionnaire raisonné de la théorie du langage, Hachette, 1979) soutient que:

En linguistique, on entend plus précisément par classe un ensemble de grandeurs substituables dans une position syntagmatique et dans un contexte donné. La classe est, en ce sens, synonyme de paradigme.

Cette définition met en évidence le côté productif de la notion de classe 
les manifestations de surface, c'est-à-dire les énoncés, sont perçues comme étant des variantes de modèles supra-organisationnels, dont l'apparition est liée à des contextes. Cette conception de la notion de classe facilite la compréhension des notions de typologie de textes et de discours dans leur spécificité et leur imbriquement.

\section{Textes et discours}

Pour mieux comprendre la signification de la diversité des typologies recensées plus loin, il faut aussi chercher à distinguer les concepts de textes et de discours.

André Petitjean pose clairement le problème (« Les typologies textuelles "), Pratiques, no 62, juin 1989) :

Pour les uns, discours et texte sont des expressions synonymiques; pour les autres, discours s'oppose à texte, le premier désignant un énoncé mis en situation et le second un mode d'organisation abstrait.

Renforçant cette distinction, Jean-Michel Adam (« Types de séquences textuelles élémentaires ", Pratiques, no 56, décembre 1987) écrit :

Un type de discours n'a pas de réalité sémiotique lorsqu'il est isolé dé son contexte, de ses rapports à d'autres discours, des situations qui le déterminent et où il a des effets. On parlerait ainsi de discours politique, de discours publicitaire, de discours littéraire, de discours scientifique, religieux, juridique ou encore journalistique.

Pour sa part, Jean-Louis Chiss (déjà cité) exprime la distinction suivante :

(Les textes) sont des ensembles d'énoncés organisés selon des règles de cohérence (tandis que les discours) sont des pratiques du langage par des sujets dans une histoire,

Il est donc possible d'en conclure que la textualité s'appréhende par une analyse syntaxique de ses composantes, telles qu'elles se manifestent dans l'énoncé, alors que le discours s'analyse par les traces que laissent les relations entretenues entre l'énoncé et l'énonciateur (ainsi que ses conditions de 
production).

Il s'ensuit que les critères de classification, et les questions qu'ils entraînent à la lecture des textes, seront différents, selon que l'on cherche à typer les textes ou les discours.

\section{Typologie des textes}

La typologie des textes s'appuie essentiellement sur le repérage de marques de surface caractéristiques dans les textes. Ces marques de surface servent de points de repère pour classer les textes à partir d'un foyer conceptuel homogène. André Petitjean ( « Les typologies textuelles », article cité) définit ainsi la notion de foyer conceptuel homogène :

Le classificateur travaille à partir d'une base typologique unique et homogène sous la forme d'un modèle abstrait, souvent déductif, qui se réalise complètement ou partiellement dans un domaine d'application donné.

En ce qui a trait à la saisie du texte source, objet qui nous préoccupe ici, nous avons relevé deux grands foyers conceptuels homogènes en typologie des textes. Ce sont les typologies par processus cognitif caractéristique et par actes de langage.

En énumérant les processus cognitifs caractéristiques, l'Allemand E. Werlich, cité par Jean-Michel Adam (« Quels types de textes? » Le francais dans le monde, no 192,1985$)$ propose cinq types textuels :

1. Le type descriptif, qui présente des arrangements dans l'espace.

2. Le type narratif, concentré sur des déroulements dans le temps.

3. Le type expositif, associé à l'analyse et à la synthèse de représentations conceptuelles.

4. Le type argumentatif, centré sur une prise de position.

5. Le type instructif, qui incite à l'action.

L'analyse de ces types est basée sur le repérage de marques de surface dans les textes: les types descriptifs sont reconnaissables en raison d'une accumulation d'imparfaits, les types narratifs offrent à lire des passés simples, les types argumentatifs ou expositifs se distinguent par la dissémination de connecteurs spécifiques (mais, puisque, dans l'argumentatif; car, parce que, 
dans l'expositif. Enfin, le type instructif sera reconnaissable par l'accumulation de verbes à l'impératif ou de verbes d'action à l'infinitif.

Pour sa part, Jean-Michel Adam (article cité) enrichit cette typologie en proposant huit types de textes, basés sur les actes de langage : asserter, convaincre, ordonner, prédire et questionner.

L'assertion est à l'origine de trois types textuels : le type narratif (1), caractérisé par l'assertion d'énoncés de faire, le .ype descriptif (2), dominé par l'assertion d'énoncés d'état, et le type explicatif (3), dans lequel l'assertion signifie expliquer des concepts et faire comprendre quelque chose à quelqu'un.

A l'acte de persuasion correspond le type argumentatif (4), tandis que l'ordre domine le texte injonctif (5). À ces cinq types qu'il qualifie de classiques, Adam ajoute trois autres types textuels, soit le type prédictif (6), manifesté dans la prophétie, l'horoscope, le bulletin météorologique, le type conversationnel (7), qu'on retrouve dans l'interview, le dialogue, et enfin, le type rhétorique (8), qui correspond au poème, à la chanson, au slogan, au titre.

Ces huit types textuels sont repérables, en surface, par l'enchaînement des énoncés suivant une superstructure syntaxique caractéristique, que l'on peut assimiler à la notion de plan. Les énoncés s'enchaînent dans un ordre dont la logique est propre à chaque type d'acte de langage. Ainsi, le texte narratif se reconnaît par la présence de la séquentialisation des étapes permettant la modification de l'état d'un sujet par l'effet des énoncés de faire cumulés dans un programme narratif. Les modèles proppien et greimassien trouvent ici toute leur utilité.

Le texte argumentatif, pour sa part, se reconnaît dans une superstructure organisationnelle ayant comme éléments la thèse et ses prémisses, l'antithèse et son argumentation et la nouvelle thèse. Le texte explicatif suit également un autre schéma organisationnel bien connu, articulé sur la séquentialisation de la mise en place d'une réalité problématique et de l'examen des causes, des conséquences et des solutions qui découlent de la description initiale de la problématique.

Les types narratifs, descriptifs, explicatifs, argumentatifs et injonctifs sont relativement bien connus et leur syntaxe discursive fait l'objet depuis longtemps de l'attention des chercheurs. Les textes prédictifs, conversationnels et rhétoriques échappent davantage à l'analyse jusqu'ici.

\section{Typologie des discours}

L'autre approche typologique dominante est basée sur la classification 
des discours. Le mode énonciatif, les intentions de communication, les conditions de production et la thématique abordée sont les principaux éléments retenus pour catégoriser les discours. On constate que la typologie des discours fait intervenir l'énonciateur dans ses relations avec le texte et son contexte, ce qui a pour conséquence que ces typologies font intervenir plusieurs foyers de critères classificatoires. En cela, elles se distinguent des typologies textuelles, basées sur un seul foyer classificatoire.

Les typologies énonciatives sont articulées sur les rapports entre l'énonciateur du discours et l'énoncé. Ces rapports s'expriment par la situation d'énonciation, laquelle se définit par le je, ici, maintenant de l'énonciateur dans le discours énoncé. Les typologies énonciatives ont pour point de départ les travaux d'Emile Benveniste, qui a proposé une typologie des discours fondée sur la présence ou l'absence, la corrélation et la densité des indicateurs linguistiques de l'instance de l'énonciation dans le discours.

Ces indicateurs justifient l'existence de deux types de discours, selon Benveniste (Problèmes de linguistique générale, I et II, Gallimard, 1966), qui correspondent à deux attitudes élocutives: l'énonciation dite personnelle, qui se manifeste par la présence explicite de l'énonciateur dans son discours, et l'autre dite historique, où l'énonciateur ne se manifeste pas.

Il est à noter que cette distinction est rejetée par le linguiste Oswald Ducrot (Dire et ne pas dire, Hermann, 1972), qui soutient qu' " il devient impossible d'admettre l'existence d'une histoire au sens de Benveniste, sinon comme l'horizon d'attente mythique de certains discours. "

Outre les modalités d'énonciation qui expriment la manière qu'a l'énonciateur de se manifester dans le discours, il faut considérer les modalités d'énoncé, qui traduisent les rapports qu'entretient l'énonciateur avec son énoncé. A la suite de D. Maingueneau (Initiation aux méthodes de l'analyse du discours, Hachette, 1976), Sophie Moirand (Situations d'écrit, Clé International, 1979, p. 14) propose de distinguer entre les modalités appréciatives, c'est-à-dire les moyens linguistiques d'exprimer l'opinion, le jugement de l'énonciateur et les modalités logiques, qui expriment sa manière de projeter l'énoncé sur l'axe du certain, du probable, du nécessaire et du virtuel.

André Petitjean ("Les typologies textuelles», article cité), pour sa part, suggère d'appeler textes objectivants les textes pauvres en indicateurs énonciatifs et textes subjectivisants les textes saturés par la présence de ces marques. 


\section{Typologie fonctionnelle}

Une autre manière prolifique d'aborder la typologie des discours est de considérer dans le discours la trace des fonctions du langage.

La typologie fonctionnelle la plus connue est celle de Roman Jakobson (Essais de linguistique générale, Minuit, 1973), basée sur les intentions de la communication. Ses variantes sont en très grand nombre. Selon Jakobson, chaque type discursif est associé à une fonction dominante du langage : référentielle, expressive, conative, phatique, métalinguistique et poétique.

Cette typologie renvoie de nouveau à l'énonciateur du discours, aux rapports qu'il entretient avec l'énoncé : le lecteur cherche le point de vue organisateur du discours, la pensée qui pense le texte et lui donne forme, en raison d'une intention dominante. Selon cette intention, l'énonciateur du discours laisse des traces linguistiques plus ou moins apparentes dans l'énoncé. L'étude des marques de l'énonciation énoncée conduit au relevé de l'intention de communication.

Autre foyer classificatoire en typologie des discours, les situations d'écrit peuvent servir de point de départ à une volonté de retracer des modèles organisationnels abstraits derrière la multitude des textes occurences, surtout en milieu administratif. Le principe de classification est ici le domaine social à partir duquel les discours sont produits. L'énonciateur individuel s'estompe ici au profit d'un énonciateur collectif dont le référent peut être une instance institutionnelle. Cet énonciateur entretient avec les discours des relations typées qui peuvent se traduire par des pratiques particulières.

De l'autre côté du miroir situationnel, on peut tenter d'énumérer des pratiques propres à certains lieux sociaux. Cette typologie présuppose que des contraintes formelles sont propres à des lieux sociaux donnés, ce qui se manifeste par des organisations discursives particulières. Ainsi, les rapports, comptes rendus, procès-verbaux, quirenvoient au monde administratif, constituent des genres d'écrits typés, que l'on apprend à reconnaître et dont l'organisation logique peut être anticipée.

Une fois posé le postulat d'une organisation discursive type liée à un lieu social ou à une pratique professionnelle particulière, il est inévitable d'aborder le contenu de ces écrits d'un même point de vue typologique. Les discours sociaux, qu'ils soient littéraires ou fonctionnels, sont ancrés dans une histoire, un continuum idéologique qui laisse des marques à plusieurs niveaux, dont au niveau thématique.

Lorsqu'on aborde une production écrite d'une période circonscrite, il n'est pas rare de voir développés un nombre limité de thèmes, qui sont des 
entités abstraites, associées à des lexèmes, et dont le potentiel de signification est exploité sous la forme de configurations discursives.

En voici la définition selon Greimas (Dictionnaire ..., op.cit., p.58), dont la réflexion est tributaire des recherches linguistiques et formalistes sur le même sujet, depuis le début du siècle :

Les configurations discursives apparaissent comme des sortes de micro-récits ayant une organisation syntactico-sémantique autonome et susceptibles de s'intégrer dans des unités discursives plus larges, en y acquérant alors des significations fonctionnelles correspondant au dispositif d'ensemble.

Si l'on prend pour exemples les thèmes suivants : l'excellence et le $21 \mathrm{e}$ siècle, on constate que ce sont des thèmes bien exploités depuis une dizaine d'années dans les écrits fonctionnels, tels les allocutions, les rapports annuels. Une lecture attentive permet de déceler l'existence de configurations discursives basées sur ces thèmes. Ces configurations se manifestent sous la forme de micro-récits types dans lesquels le sujet est conjoint ou disjoint avec la valeur dominante et selon l'état du sujet, les événements s'enchaînent suivant un ordre syntaxique prédéfini.

Les configurations discursives sont en quelque sorte des prêts-à-penser dans nos sociétés: quiconque participe de l'univers des communications professionnelles les repère et les utilise dans différents contextes.

La reconnaissance des configurations discursives ouvre la voie à un inventaire de ces organisations discursives dans un univers culturel donné, comme le soulignait le Groupe d'Entrevernes dans Analyse sémiotique des textes (Presses de l'Université de Lyon, 1979).

Cette démarche apparaît d'autant plus pertinente dans l'enseignement du résumé fonctionnel qu'elle donne une possibilité d'extraire des productions écrites des pans complets, déplaçables et analysables comme tels. Le résumé de ces segments est facilité par le caractère répétitif de leur organisation syntaxique et de leur investissement sémantique.

\section{Typologie et résumés}

L'examen de ces théories typologiques avait pour but d'application la compréhension des mécanismes utiles de saisie d'un texte source que l'on cherche à résumer dans un contexte de rédaction professionnelle. Le résumé exige du résumeur une capacité d'appréhension du texte écrit de force égale à 
sa capacité d'en produire une version abrégée qui tient compte d'une situation de communication particulière.

Étant posées ces exigences dans la rédaction de résumés, il apparaît que les typologies des textes et des discours ne sont pas dissociables lorsqu'il s'agit d'élaborer une méthode d'enseignement des techniques du résumé. L'acte de résumer engage le résumeur à saisir la production écrite comme textualité et comme manifestation discursive.

Sur le plan textuel, le texte source s'appréhende avec plus d'aisance si la séquentialisation des énoncés renvoie à des patterns reconnaissables.

Que ce soit par processus cognitif caractéristique ou par acte de langage, la typologie textuelle permet de ramener les productions écrites à un certain nombre de structures syntaxiques caractéristiques et répétitives, qui facilitent grandement la lecture analytique des textes. Le repérage de ces structures favorise la saisie du sujet de la communication, le point de vue de l'énonciateur et l'articulation du sujet sur un certain nombre d'idées principales.

Les structures syntaxiques associées aux types textuels se lisent comme des organisations logiques, c'est-à-dire des mécanismes de mise en relation logique des idées investies dans les structures. La reconnaissance des structures textuelles types renforce la capacité du résumeur de saisir avec justesse la pensée logique qui s'énonce dans le texte.

Quant à l'apprentissage des typologies discursives, il permet de reconnaître qu'explicite ou implicite, l'énonciateur, individuel ou social, est toujours présent dans le texte et qu'il oriente ce texte selon ses intentions.

L'enjeu est ici d'établir la différence entre un type de texte bien marqué sur le plan énonciatif et un autre où ces traces sont effacées. Dans tous les cas, l'objectif prioritaire est de repérer la ou les voix qui s'expriment dans tout discours : il faut ici comprendre que tout énoncé présuppose toujours quelqu'un qui l'énonce.

Les critères de classification derrière les typologies discursives servent à mettre en évidence les multiples détours que prend l'énonciateur pour envahir son discours. Les typologies s'additionnent, se complémentent et font ressortir un ensemble de questions indispensables pour aborder le résumé de manière productive et faire échec à une perception éclatée de la textualité : comment comprendre une infinité de textes disparates, uniques et sans liens les uns avec les autres?

Il est donc utile de faire connaît re les patterns de communication, particulièrement dans un contexte de production fonctionnelle, de rédaction professionnelle. À cet égard, des modèles typologiques composites se révèlent 
des plus pertinents, prenant en compte la dimension texte et la dimension discours des énoncés.

Les typologies étant tributaires des environnements qu'elles cherchent à décrire, elles évoluent et les critères sur lesquelles elles s'appuient sont constamment réexaminés pour fins de discussion. Les typologies sont également toujours incomplètes, a work in progress, dirait-on en milieu théâtral. C'est, entre autres, ce qui les rend ntéressantes dans la poursuite d'une réflexion sur la caractérisation des écrits fonctionnels courants, dont le résumé est une composante des plus significatives. 


\section{Références}

Jean-Michel Adam. Quels types de textes? Le français dans le mond, no. 192, 1985.

Jean-Michel Adam. Types de séquences textuelles élémentaires. Practiques, no. 56, décembre 1987.

Emile Benveniste. Problèmes de linguistique générale. I et II, Gallimard, 1966.

Michel Charolles et André Petitjean. L'Activité résumante. Université de Metz, 1992.

Michel Charolles et André Petitjean. Le résumé de texte, aspects linguistiques, sémiotiques, psycholinguistiques et automatiques. Klincksieck, 1992.

Jean-Louis Chiss. Malaise dans la classification. Langue française, no. 74, mai 1987.

J. Courtés, A. J. Greimas. Sémiotique, Dictionnaire raisonné de la théorie du langage. Hachette, 1979.

Oswald Ducrot. Dire et ne pas dire. Hermann, 1972.

Oswald Durcot et Tzvetan Todorov. Dictionnaire encyclopédique des sciences du langage. Seuil, 1972.

Group d'Entrevernes. Analyse sémiotique des textes. Presses de l'Université de Lyon, 1979.

Roman Jakobson. Essais de linguistique générale. Minuit, 1973.

D. Maingueneau. Initiation aux méthodes de l'analyse du discours. Hachette, 1976.

Sophie Moirand. Situations d'écrit. Clé International, 1979.

André Petitjean. Les typologies textuelles. Practiques, no. 62, juin 1989.

M.Riffaterre. Systéme d'un genre descriptif. Poétique, no. 9, 1972.

Jean-Paul Simard. Guide du savoir-écrire. Editions de l'homme, 1984.

Tzvetan Todorov. Introduction à la littérature fantasique. Seuil, 1970.

Robert Tremblay. L'écritoire. McGraw-Hill, 1991. 
